\title{
Technologie und Alter in Japan
}

\author{
Yasuyuki Gondo
}

Der Anteil älterer Menschen an der Bevölkerung hat in den letzten Jahrzehnten in Japan zugenommen. Dieser signifikante demographische Wandel hat Unternehmen neue geschäftliche Möglichkeiten eröffnet. Speziell für ältere Menschen sind innovative Technologien und Dienstleistungen entwickelt worden, von denen einige bereits angewendet werden. Die weitere, zu erwartende technologische Revolution scheint für die künftige Gesellschaft mit extrem vielen alten Menschen ein erfolgreiches Altern zu versprechen. Dieser Bericht vermittelt einen Überblick über Technologien, die derzeit von älteren Menschen angewandt werden. Er stellt ferner einige Beispiele für neue Technologien vor, die in Japan entwickelt werden.

\section{Einführung}

Japan ist eines der Länder, in denen die Alterung der Gesellschaft am stärksten fortgeschritten ist (Muramatsu und Akiyama, 2011). Daher können auch andere Länder aus den japanischen Erfahrungen nützliche Lehren ziehen. Insbesondere im technologischen Bereich sind viele operative Dienstleistungen bereits eingeführt worden oder befinden sich derzeit in der Entwicklung. Um diesen Themenkomplex anzugehen, habe ich Technologien für ältere Menschen in drei funktionelle Kategorien unterteilt: Kompensation, Kommunikation und Gesundheitsförderung. Mit Kompensation ist hier die Unterstützung oder Wiedererlangung einer Funktion durch den Einsatz von Technologien, wie z. B. Hörgeräten, gemeint. Mit Kommunikation werden zwischenmenschliche Bindungen und die Verbesserung des Wohlbefindens bezeichnet. Gesundheitsförderung bedeutet die Erhaltung der Gesundheit durch Einsatz von Kommunikationstechnologien, z. B. bei der Aktivitätsüberwachung mithilfe von Smartwatches.

\section{Kompensationstechnologie}

Eine Umfrage ergab, dass ältere Japaner im gleichen Umfang über Hörprobleme klagen wie ältere Menschen in Europa (etwa $10 \%$ ). Allerdings gibt es in Japan weniger Träger von Hörgeräten (14 \%) als in westlichen Ländern (24 - $41 \%$ ) (JapanTrak 2012). Während im oder hinter dem Ohr getragene Geräte nicht populär sind, werden andere Arten von Geräten wie Knochenleitungshörgeräte und deren Anwendung bei Knochenleitungstelefonen immer beliebter. Es werden jedoch noch weitere einzigartige Maschinen auf den Markt gebracht. Das beliebteste Gerät ist ein Lautsprechersystem für Fernsehgeräte, das es älteren Menschen ermöglicht, den TV-Ton in ihrer Nähe zu hören, ohne dafür die Lautstärke des Fernsehers erhöhen zu müssen. 
Im Bereich der Rundfunktechnik könnte ein Umwandlungssystem für die Sprechgeschwindigkeit, das in Echtzeit funktioniert, in der Zukunft mehr Beachtung finden. Ein solches System wurde vor mehr als 10 Jahren entwickelt. Das System wurde vom NHK Broadcasting Culture Research Institute erfunden und es wurden Radios auf den Markt gebracht, die mit ihm ausgerüstet sind (https://www.nhk.or.jp/strl/publica/bt/en/pr0013.pdf). Dieses Produkt passt buchstäblich zu den Anforderungen der Zeit. Seit den Anfängen im Jahr 1989 sind ältere Menschen die wichtigste Hörergruppe des vom Sender NHK ausgestrahlten Nachtprogramms. Derzeit hat NHK viele pensionierte DJs engagiert (im Alter von 49 bis 74 Jahren; Durchschnittsalter: 64 Jahre) und strahlt besonders Inhalte aus, die ältere Hörer gerne mögen. Dies ist ebenfalls ein Beleg dafür, dass die latente Nachfrage nach Hörhilfen bei älteren Menschen hoch ist. Ein wesentlicher Grund für die Ablehnung von Hörgeräten ist das Unbehagen, das von schlecht eingestellten Geräten verursacht wird. Es werden jedoch auch anspruchsvollere und integrierte Dienstleistungen im Bereich der Hörhilfe benötigt.

Hilfsmittel zur Kompensation von Mobilitätseinschränkungen sind für ein selbstbestimmtes Leben unverzichtbar. Wenn man in japanischen Innenstädten einkaufen geht, sind ältere Frauen mit Rollatoren nicht zu übersehen (in Japan auch gerne „Silber-Autos“ genannt). Für dieses Hilfsmittel gibt es eine Vielzahl bunter Dekorationen zu kaufen. „Silber-Autos“ verfügen in der Regel über Einkaufskörbe und Sitzbänke, um sich vorübergehend auszuruhen.

Bei gebrechlicheren Menschen sind Fahrzeuge mit Antrieb sehr beliebt. Die ersten kommerziellen Produkte kamen in den 1980er Jahren in die Geschäfte. Gegenwärtig werden zwei Arten von Fahrzeugen verkauft: mit Joystick und mit Griff-Bedienung. Interessanterweise ist das Handelsvolumen seit dem Spitzenjahr 2002 (33.477 Stück) bis 2014 (19.438 Stück) zurückgegangen. Einer der plausiblen Erklärungen für die schwindende Popularität ist die zunehmende Verbreitung der Redensart „Wer rastet, der rostet“ in Japan. Nicht nur ältere Menschen, sondern auch Familienmitglieder als die möglichen Betreuer machen sich in Japan Sorgen, dass die Abhängigkeit von diesen bequemen Geräten zu einem stärkeren Funktionsverlust führen kann. Kürzlich sind Rollstühle in Kombination mit fahrradähnlichen Pedalen auf den Markt gekommen (http://www.starlinxpedalchair.com/history.html). Der Vorteil dieses Fahrzeugtyps ist, dass Benutzer sich auch dann mit den eigenen Beinen fortbewegen können, wenn sie im Rollstuhl sitzen. Es sind auch Roboter mit modernster Technologie entwickelt worden, die verlorene Funktionen ersetzen (http://www.cyberdyne.jp/english/products/HAL/index.html). Die Bereitstellung eines Mindestmaßes an Unterstützung für abnehmende Funktionen könnte ein Trend bei zukünftigen Kompensationstechnologien sein.

Für die Überwindung kurzer Entfernungen außerhalb von Wohnanlagen sind elektrische Kleinstfahrzeuge führende Kandidaten. Diese Fahrzeuge bieten den Vorteil geringer Emissionen und eines komfortablen Gebrauchs. Derzeit ist jedoch für ihre Nutzung ein Führerschein erforderlich. Die 
japanische Regierung hat für Fahrer ab 75 Jahren eine Prüfung zur Führerscheinverlängerung eingeführt, um die Anzahl an Unfällen zu verringern. Wenn man bedenkt, wer die Hauptnutzer dieser Fahrzeuge sind, wird die Verlängerung der Fahrerlaubnis in Zukunft ein großes Problem darstellen. Die Implementierung selbstfahrender Systeme wird daher bei der künftigen Entwicklung unverzichtbar sein.

\section{Kommunikation}

Ein weiteres Thema, das in Japan eine Rolle spielt, ist, dass immer mehr ältere Menschen alleine leben. Die japanische Regierung schätzt, dass 2015 rund 6,0 Mio. ältere Menschen allein leben und dass sich deren Zahl im Jahr 2035 voraussichtlich auf 7,6 Mio. erhöhen wird. Eine 2005 ausgestrahlte TV-Dokumentation zu „kodoku shi“ - dem Tod eines Menschen, der allein lebt, zu Hause stirbt und dies lange Zeit unbemerkt bleibt - hat die japanische Gesellschaft beeinflusst. Das Weißbuch über die alternde Gesellschaft im Jahr 2015 berichtete, dass $45 \%$ der allein lebenden älteren Menschen glauben, dass „kodoku shi“ ein Problem ist, von dem sie betroffen sein können. Es wird davon ausgegangen, dass eine Kommunikationsvorrichtung, die ältere Menschen mit ihrer Familie oder der Gesellschaft verbindet, soziale Isolation verhindern und Fälle von „kodoku shi“ reduzieren kann. In Japan gibt es eine lange Geschichte der Verwendung von Technologien für die Lösung dieses Problems. In den 1970er Jahren wurden Notrufsysteme für ältere Menschen entwickelt. Hierbei handelt es sich um ein aktives Kommunikationssystem, das mithilfe von anhängerartigen drahtlosen Endgeräten oder Notruftasten an der Wand über Notfälle informiert. Im Jahr 2000 stellten mehr als $95 \%$ der örtlichen Behörden einen solchen Dienst in irgendeiner Weise zur Verfügung. Jedoch wurde in mehreren Artikeln berichtet, dass weniger als $10 \%$ der Seniorenhaushalte die Systeme auch tatsächlich nutzten; ältere Menschen zögern häufig, das System zu verwenden, auch wenn sie es haben.

Das „Mimamori“ (in etwa „Aufpasser“) genannte Hotline-System, bei dem ein elektrischer Wasserkocher verwendet wird, um die Aktivitäten älterer Menschen zu überwachen, wurde zum ersten Mal im Jahr 2001 kommerziell mit einem Produkt des täglichen Lebens eingesetzt. Dieser Dienst wurde von einem Thermosflaschen-Hersteller entwickelt (http://www.zojirushiworld.com/activities/amenities.html). Das System überwacht die Nutzung elektrischer Wasserkocher durch die Bewohner und ermöglicht somit eine Überwachung älterer Menschen mittels passiver Kommunikation. Angesichts der Tatsache, dass ältere Menschen eher dazu neigen, aktive Notfallkommunikationsgeräte nur zögerlich zu verwenden, war die passive Kommunikation damals eine hervorragende Idee. Leider sank die Zahl der Nutzer von 7.600 beim Start auf heute nur noch 3.600. Das Konzept der passiven Kommunikation hat jedoch überlebt und wird nun in moderneren Vorrichtungen verwendet, wie bei der Überwachung der Strom- oder Stadtgas-Nutzung, der Bewegungserkennung oder für biologische Funktionen wie Atemerkennungssensoren im Haus. In jüngster Zeit wurden von verschiedenen Unternehmen Prototypen humanoider 
Kommunikationsroboter vorgestellt, die Kommunikationsfunktionen umsetzen. Die Firmen planen, Roboter als wichtigste Schnittstellen für umfassende Dienste von der Überwachung bis hin zur Gesundheitsförderung einzusetzen. Wegen Problemen mit der Zuverlässigkeit befinden sich humanoide Roboter, die zur Überwachung und Gesundheitsförderung eingesetzt werden, in einer Testphase und sind noch nicht im Handel erhältlich.

Derzeit ist das kommerziell erfolgreichste Produkt im Kommunikationsbereich ein Roboter mit dem Namen Paro. Paro ist ein Roboter, der einem Sattelrobbenbaby nachempfunden ist und der heilende und therapeutische Effekte haben soll (http://www.parorobots.com/). Auch wenn keine Sprachkommunikation möglich ist, wurde eine heilende Wirkung wissenschaftlich nachgewiesen. Viele Pflegeheime in Japan und anderen Ländern haben bereits begonnen, Paro einzusetzen, hauptsächlich zur psychischen Stabilisierung von Demenzkranken. Telenoid (http://www.geminoid.jp/projects/kibans/res/Telenoid-resources.html) ist ein ebenfalls in Japan erfundener Kommunikationsroboter. In der Erprobungsphase in Dänemark zeigen demente Menschen interessanterweise ein inniges Verhältnis zu diesem ungewöhnlichen Roboter.

\section{Gesundheitsförderung}

Der Bereich der Gesundheitsförderung ist ein wachsendes technologisches Segment, nicht nur für ältere Menschen. In diesem Jahr begann eine große epidemiologische Studie mit 1.000 älteren Menschen zur Inzidenz von Demenz unter Verwendung eines Armband-Aktivitätssensors. In diesem Projekt werden tägliche Aktivitäten, Schlaf sowie die Häufigkeit von Gesprächen mithilfe von Armbändern überwacht, und es wird die Korrelation mit den Beta-Amyloid-Spiegeln im Gehirn untersucht (http://www.pcworld.com/article/2971792/gadgets/toshiba-rolls-out-activity-trackers-forseniors.html).

Neben innovativen Überwachungsvorrichtungen werden auch gängige Technologien neu überdacht. Vor einigen Jahren berichteten die Medien, dass ältere Menschen allmählich die Mehrheit der Besucher von Vergnügungszentren bilden (http://edition.cnn.com/2012/02/08/world/asia/japan-oldergamers). Die „neue“ ältere Generation ist eher vertraut mit Spielautomaten und TV-Spielen als die Senioren früherer Tage. Spielautomatenhersteller haben ihre Spiele so verändert, dass sie auch als Rehabilitationshilfe und zur Gesundheitsförderung dienen. „Drum Master“ ist beispielsweise ein Spiel, bei dem das richtige Timing geprüft wird, indem Trommeln im Takt bestimmter Lieder geschlagen werden. In den modifizierten Versionen aus dem Jahr 2004 wurde zu Liedern übergegangen, die älteren Menschen eher vertraut sind. Darüber hinaus umfassten sie Unterstützungsmechanismen zum Halten der Trommelstöcke, tiefere Trommelpositionen für Rollstühle und einfachere Kriterien für das Timing beim Schlagen der Trommel. Im selben Jahr gründete dieses Unternehmen Pflegeeinrichtungen, die mit Spielautomaten ausgestattet wurden. Derzeit werden andere Arten von Spielautomaten verkauft, die zum körperlichen und geistigen Training älterer Menschen dienen. Es wird davon ausgegangen, dass diese Geräte eine Alternative zu 
Rehabilitationsfachkräften darstellen und die Kosten für Pflege und Rehabilitation reduzieren können.

\section{Schlussfolgerung}

In den letzten Jahrzehnten wurden zahlreiche Technologien speziell für ältere Menschen entwickelt und verkauft. Manche sind immer noch im Einsatz und werden weiterentwickelt, andere sind heute nicht mehr so beliebt. Der Autor vermutet, dass drei Faktoren der Schlüssel zur Popularität sind: möglichst komfortabler Gebrauch, minimale Unterstützung, die keinen weiteren Funktionsverlust verursacht, sowie auf Höhe der Zeit sein. Ältere Menschen wägen Nutzen und Risiken des Einsatzes von Technologien sehr sorgfältig ab. In diesem Jahr hat eine Mobiltelefongesellschaft begonnen, einen humanoiden Kommunikationsroboter (http://www.softbank.jp/en/robot/) direkt an Verbraucher zu verkaufen. Wie bereits erwähnt, wird davon ausgegangen, dass Kommunikationsroboter eine Schnittstelle für verschiedene Dienste bilden werden, die älteren Menschen zur Verfügung gestellt werden. Die japanische Regierung hat eine nationale Roboterstrategie festgelegt (http://www.meti.go.jp/english/press/2015/pdf/0123_01b.pdf) und fördert massiv die zukünftige industrielle Entwicklung. Wenn alle notwendigen Bedürfnisse sowie Kompensation, Kommunikation und Gesundheitsförderung in Roboter integriert werden, wird dies eine echte technische Revolution sein und Gesellschaften mit extrem vielen alten Menschen dabei helfen, die Herausforderungen erfolgreich zu meistern.

\section{Literatur}

Muramatsu, N. \& Akiyama, H. (2011) Japan: Super-Aging Society Preparing for the Future. The Gerontologist, $51 / 4,425-432$.

Hougaard S., Ruf S. \& Egger C. (2013) EuroTrak + JapanTrak 2012: Societal and Personal Benefits of Hearing Rehabilitation with Hearing Aids - http://www.hearingreview.com/2013/03/eurotrak-japantrak-2012societal-and-personal-benefits-of-hearing-rehabilitation-with-hearing-aids/\#sthash.9spe5nAY.dpuf.

\section{Danksagung:}

Die über das Internet zugänglichen englischen Informationen wurden hauptsächlich für nichtjapanischsprachige Leser zur Verfügung gestellt. Dieser Artikel wurde mit Fördermitteln (Nr. 263101040) aus dem japanischen Ministerium für Bildung, Kultur, Sport, Wissenschaft und Technologie unterstützt. Das Durchschnittsalter von Rundfunk-DJs wurde freundlicherweise zur Verfügung gestellt von Fr. Kazuko Yasumura.

Autor: Prof. Dr. Yasuyuki Gondo ist Associate Professor an der Universität Osaka, Graduate School of Human Sciences and Clinical Thanatology and Geriatric Behavioral Science.

Kontakt: ygondo@hus.osaka-u.ac.jp. 